\title{
Sepsis neonatal por Streptococcus Grupo B y Bacterias gram negativas: prevalencia y riesgo de meningitis
}

\author{
LUIS A. MENDOZA T. ${ }^{1}$, MIGUEL OSORIO R. ${ }^{2}$, MARTHA ARIAS G. ${ }^{3}$, LAURA MENDOZA T. ${ }^{4}$ \\ 1. Pediatra Neonatólogo. Fundación Hospital San José de Buga. Unidad Central del Valle, Tuluá, Colombia. \\ 2. Pediatra Neonatólogo. Universidad del Valle, Colombia. \\ 3. Pediatra. Fundación Hospital San José de Buga, Colombia. \\ 4. Estudiante de Medicina. Unidad Central del Valle, Tuluá, Colombia.
}

\begin{abstract}
Sepsis neonatal Group B Streptococcus and gram negative bacteria: prevalence and risk of meningitis

Introduction: Sepsis and meningitis are major causes of neonatal morbidity and mortality. Objective: To identify the prevalence and risk of meningitis in neonates with sepsis due to group B Streptococcus (GBS) and gram-negative bacteria (GNB), and to compare risk factors, clinical and laboratory characteristics. Patients and Method: Cross-sectional study of 30 infants with infections due to GBS and 41 due to BGN. Data were analyzed by Stata ${ }^{\circ} 11.0$, using measures of central tendency and dispersion, according to their distribution, to compare differences. Categorical variables were compared using $\chi^{2}$ test or Fisher's Exact Test and associations using prevalence ratios (PR) and odds ratio (OR) with $95 \%$ confidence interval. A level of probability of $<0.05$ was used as a significance criterion. Results: The prevalence of meningitis among infants with sepsis was $16.9 \%$ (95\% CI: $7.5-26.3 \%) .33 .3 \%$ (10/30) of sepsis due to GBS was associated with meningitis, while $4.9 \%(2 / 40)$ was associated with GNB, finding a clear connection between sepsis and meningitis in neonates with GBS (OR: 9.5; CI 95\%:1.7-94.3). 80\% (CI 95\%: 44,4-97,5\%) of cases of meningitis associated with GBS sepsis occurred in infants older than 72 hours. Conclusion: Meningitis was more common in infants with GBS sepsis, mainly in cases of late-onset sepsis. The association between GNB sepsis and meningitis was less frequent in early sepsis, and was not found in late sepsis.

(Key words: Sepsis, meningitis, neonatal, GBS, Gram-negative bacteria).

Rev Chil Pediatr 2013; 84 (5): 513-521
\end{abstract}

\section{RESUMEN}

Introducción: La sepsis y meningitis son importante causa de morbi-mortalidad neonatal. Objetivo: Identificar la prevalencia y riesgo de meningitis en neonatos con sepsis por Streptococcus del grupo B (SGB) y bacterias Gram negativas (BGN), además de comparar factores de riesgo, características clínicas y de laboratorio.

Recibido el 15 de agosto de 2012, devuelto para corregir el 7 de enero de 2013, segunda versión 17 de enero de 2013, tercera versión 16 de abril de 2013, aceptado para publicación el 22 de agosto de 2013.

Este trabajo cumple con los requisitos sobre consentimiento /asentimiento informado, comité de ética, financiamiento, estudios animales y sobre la ausencia de conflictos de intereses según corresponda.

Correspondencia a:

Luis Alfonso Mendoza Tascón

E-mail: lamendozat@gmail.com 
Pacientes y Método: Estudio de corte transversal con 30 neonatos con SGB y 41 con BGN. Los datos fueron analizados en Stata ${ }^{\circledR}$ 11.0, empleando medidas de tendencia central y dispersión de acuerdo a su distribución para comparar diferencias. Las variables categóricas fueron comparadas con prueba de $\chi^{2}$ o Exacta de Fisher y las asociaciones mediante razones de prevalencia (RP) y odds ratio (OR) con su intervalo de confianza de 95\%. Se empleó un nivel de probabilidad $<0,05$ como criterio de significancia. Resultados: La prevalencia de meningitis entre neonatos con sepsis fue 16,9\% (IC 95\%: 7,5-26,3\%). El 33,3\% (10/30) de sepsis por SGB se asoció a meningitis, mientras 4,9\% (2/40) a sepsis por BGN, hallándose una asociación entre sepsis y meningitis en neonatos con SGB (OR: 9,5; IC 95\%: 1,7-94,3). El 80\% (IC 95\%: 44,4-97,5\%) de casos de meningitis asociada a sepsis por SGB ocurrió en neonatos mayores de 72 h de vida. Conclusión: La meningitis fue más frecuente en neonatos con sepsis por SGB, principalmente en casos de sepsis tardía. La asociación entre sepsis por BGN y meningitis fue menos frecuente en sepsis temprana, y no se halló en sepsis tardía.

(Palabras clave: Sepsis, meningitis, neonato, Streptococcus del grupo B, bacterias Gram negativas).

Rev Chil Pediatr 2013; 84 (5): 513-521

\section{Introducción}

Con el advenimiento de la profilaxis antibiótica, la epidemiología de la sepsis neonatal ha cambiado en algunos países ${ }^{1,2}$. Se estima que a nivel mundial $20-30 \%$ de todas las mujeres embarazadas son portadoras de $\mathrm{SGB}^{3}$. La profilaxis antibiótica intraparto ha demostrado ser eficaz para la prevención del SGB de inicio precoz, sin embargo, el uso de antibióticos de amplio espectro ha traído como problema de gran preocupación la aparición y el incremento de resistencia a los antibióticos en las unidades de cuidado intensivo neonatal como la nuestra ${ }^{4}$.

Para nuestro país el SGB sigue siendo una causa importante de sepsis en el recién nacido. Sin embargo, otras bacterias, como la Escherichia coli (E. coli), también son importante causa de infección en el período neonatal ${ }^{5-7}$, asociado a uso creciente de antibióticos maternos durante el parto ${ }^{8}$.

La incidencia de sepsis neonatal está alrededor de 23/1.000 nacidos vivos (nv) y la meningitis 3/1.000 nv ${ }^{9}$. La sepsis temprana por SGB alcanza un $31,5 \%$ y por BGN $17,8 \%$, donde la E. coli representa el $9 \%$. Mientras tanto, en la sepsis tardía, el SGB representa el 6,6\% y las BGN el 19,3\%, donde las predominantes son las enterobacterias $(8,9 \%)$ y la $E$. coli $(6,8 \%)^{10}$.

Los objetivos de este trabajo fueron identificar la prevalencia y el riesgo de meningitis entre los neonatos con sepsis por SGB y BGN, además, de comparar los factores de riesgo, las características clínicas y de laboratorio.

\section{Pacientes y Método}

Estudio de corte transversal entre neonatos con sepsis por SGB y BGN, que fueron hospitalizados en la Unidad de Cuidados Intensivos de la Fundación Hospital San José de Buga, entre 2005-2012.

Se definió sepsis y meningitis a la manifestación clínica de sepsis acompañada del aislamiento de SGB o BGN en sangre y/o líquido cefalorraquídeo en un neonato de hasta 28 días de vida post-natal ${ }^{11,12}$.

En el trabajo se incluyeron neonatos de 24 y más semanas de edad gestacional al nacer, con un peso de $500 \mathrm{~g}$ o más al nacer, con una edad cronológica de 0 a 28 días de vida o 44 semanas de edad gestacional corregida, si se trata de un prematuro. Se excluyeron neonatos con asfixia perinatal, trisomías y malformaciones congénitas mayores.

Se incluyó todos los neonatos con aislamiento de un SGB y todos los neonatos con aislamiento de una BGN en sangre, que cumplieron con los criterios de inclusión y exclusión. Para efectos del análisis, se consideró por un lado al neonato expuesto a sepsis por SGB y por el otro al neonato expuesto a sepsis por BGN. Se seleccionaron 30 neonatos con diagnóstico de sepsis por SGB y 41 neonatos con diagnóstico de sepsis por BGN.

Con el tamaño de muestra del estudio, 30 casos de sepsis por SGB y 41 casos de sepsis por BGN y una prevalencia hallada de meningitis de 33,3\% para sepsis por SGB y de 4,9\% para BGN, para un nivel de confianza de $95 \%$, 
un efecto del diseño de $1 \%$, la precisión absoluta o máximo error absoluto de cada una de los tamaños de muestra fue de 1\% (Epidat 3.1).

El resultado de interés del estudio es la prevalencia y determinación del riesgo de meningitis en neonatos con sepsis por SGB y por BGN.

Se analizaron variables maternas y neonatales incluyendo las demográficas. Entre las variables maternas se evaluó edad al momento del parto, paridad, ruptura prematura y prolongada de membranas ovulares, duración de la ruptura de las membranas, fiebre en el período perinatal, corioamnionitis, factores asociados a infección neonatal por SGB agrupados e infección de vías urinarias. Las variables neonatales incluyeron peso al nacer, edad gestacional al nacer, edad al ingreso hospitalario, sexo, vía de nacimiento, hospitalizaciones previas, fiebre y manifestaciones clínicas agrupadas por sistemas. El laboratorio incluyó hemograma, proteína $\mathrm{C}$ reactiva (PCR), estudio de orina y líquido cefalorraquídeo, electrolitos, bilirrubinas, estudios de imágenes, diagnóstico de bacterias aisladas, morbilidad y mortalidad.

Análisis estadístico: Los análisis estadísticos se realizaron con Stata ${ }^{\circledR}$ 11.0. Las variables categóricas, nominales $\mathrm{u}$ ordinales se presentan en tablas de frecuencias con sus respectivos porcentajes. Las variables continuas se expresaron como promedios y su desviación estándar (DE) o medianas con su rango intercuartil (RI) y se evaluaron empleando la prueba t-test no pareado o Wilcoxon rank-sum (Prueba de Mann-Whitney) acordando su distribución para comparar diferencias. Algunas variables maternas (factores de riesgo para infección neonatal por SGB) y neonatales (manifestaciones clínicas) se agruparon para su análisis. Las variables categóricas fueron comparadas con la prueba de $\chi^{2}$ o prueba Exacta de Fisher. Cuando ambas variables fueron cualitativas se realizaron tablas de contingencia, a partir de las cuales se estudiaron si las dos variables eran o no independientes. La independencia entre las variables se evaluó mediante el test de $\chi^{2}$ de Pearson. Se determino la prevalencia de meningitis para neonatos con sepsis por SGB y por BGN para todas las edades cronológicas, así como para la sepsis temprana y tardía por ambos tipos de bacterias. Para estimar la asociación entre sepsis por SGB y BGN con meningitis, se empleo la RP y el OR con sus respectivos intervalo de confianza del 95\% (IC 95\%).

Para la realización de este trabajo se obtuvo la aprobación del Comité de Ética del Hospital.

\section{Resultados}

Se compararon 30 casos de sepsis por SGB y 41 casos de sepsis por BGN. En las primeras $72 \mathrm{~h}$ de vida ocurrieron el 40\% (n: 12) de los casos de infección por SGB y el 36,6\% (n: 15) de los casos por BGN.

Entre los casos de sepsis por SGB, las madres tuvieron más antecedente de parto prematuro espontáneo o por ruptura prematura de membranas. Se halló diferencia estadísticamente significativa entre baja paridad, factores de riesgo para infección por SGB maternos e infección neonatal por SGB $(\mathrm{p}<0,05)$. Por otro lado, los neonatos con infección por SGB, tuvieron mayor peso al nacer, mayor edad gestacional y la mayoría nacieron por vía vaginal, diferencias estadísticamente significativas $(\mathrm{p}<0,05)$. El 22\% de los neonatos con infecciones por BGN fueron menores de 37 semanas de gestación, mientras esto ocurrió en el 10\% de los neonatos con infección por SGB $(\mathrm{p}>0,05)$. El bajo peso al nacer $(<2.500 \mathrm{~g})$ ocurrió en mayor proporción para neonatos con sepsis por BGN que por SGB, diferencia que fue estadísticamente significativa $(p<, 05)$. El sexo masculino fue el más frecuente entre los neonatos del estudio, tabla 1.

Hubo una mayor proporción de manifestaciones clínicas entre los neonatos con infección por SGB, que entre los que presentaron infecciones por BGN. Sin embargo, sólo la fiebre y las manifestaciones respiratorias fueron significativamente mayores en neonatos con sepsis y meningitis por SGB $(\mathrm{p}<0,05)$, tabla 2 . En cuanto a los laboratorios, a pesar de que no se halló diferencia estadísticamente significativa para los componentes del hemograma, ni del líquido cefalorraquídeo (LCR), se halló un mayor número de leucocitos y neutrófilos en sangre y LCR para las infecciones por BGN. 
Tabla 1. Características maternas y neonatales

\begin{tabular}{|c|c|c|c|c|}
\hline Variable & Medida & SGB $\mathbf{n}: \mathbf{3 0}$ & BGN n: 41 & $\mathbf{p}$ \\
\hline \multicolumn{5}{|l|}{ Materna } \\
\hline Edad (años) & Promedio (DE) & $21,9( \pm 6,4)$ & $23,8( \pm 5,8)$ & 0,308 \\
\hline Paridad & Mediana (RI) & $1(1-2)$ & $2(2-3)$ & $<0,01$ \\
\hline Control prenatal con $\geq 5$ visitas & n (\%) & $27(90 \%)$ & $33(80,5 \%)$ & 0,274 \\
\hline Ruptura prematura de membranas & n (\%) & $4(13,3 \%)$ & $1(2,4 \%)$ & 0,076 \\
\hline Fiebre perinatal & $n(\%)$ & $4(13,3 \%)$ & $2(4,8 \%)$ & 0,206 \\
\hline Corioamnionitis & $n(\%)$ & $1(3,3 \%)$ & $1(2,4 \%)$ & 0,822 \\
\hline Infección urinaria en embarazo & n (\%) & $6(20 \%)$ & $4(9,8 \%)$ & 0,220 \\
\hline $\begin{array}{l}\text { Antecedente de parto prematuro o por } \\
\text { ruptura prematura de membranas }\end{array}$ & n (\%) & $4(13,3 \%)$ & 0 & 0,016 \\
\hline Factores de riesgo para SGB agrupados & n (\%) & $9(30 \%)$ & $3(7,3 \%)$ & 0,012 \\
\hline \multicolumn{5}{|l|}{ Neonatal } \\
\hline Edad (días) & Promedio (DE) & $11,1( \pm 10,3)$ & $11,6( \pm 11,3)$ & 0,853 \\
\hline Edad gestacional (semana) & Promedio (DE) & $38,7( \pm 1,7)$ & $37,6( \pm 3,5)$ & 0,049 \\
\hline Edad gestacional $<37$ sem & n (\%) & $3(10 \%)$ & $9(22 \%)$ & 0,184 \\
\hline Peso al nacer $(G)$ & Mediana (RI) & $3.186(2.900-3.420)$ & $2.850(2.600-3.200)$ & 0,036 \\
\hline Peso al nacer $<2.500 \mathrm{~g}$ & n (\%) & $1(3,3 \%)$ & $8(19,5 \%)$ & 0,043 \\
\hline Sexo masculino & n (\%) & $19(63,3 \%)$ & $30(73,2 \%)$ & 0,376 \\
\hline Nacimiento por vagina & n (\%) & $28(93,3 \%)$ & $24(58,5 \%)$ & $<0,01$ \\
\hline Hospitalización previa & n (\%) & $4(13,3 \%)$ & $3(7,3 \%)$ & 0,400 \\
\hline
\end{tabular}

SGB: Streptococcus del grupo B. BGN: Bacteria Gram negativas. DE: Desviación estándar. RI: Rango intercuartil. Factores de riesgo para SGB: Parto prematuro, antecedente de hermano con sepsis por SGB, madre febril o con corioamnionitis, madre portadora de SGB, ruptura prolongada de membranas en madre con SGB.

Para la sepsis por SGB encontramos un valor de leucocitos en sangre por debajo de $4.000 /$ $\mathrm{mm}^{3}$ y por encima de $15.000 / \mathrm{mm}^{3}$ en $45 \%$ de casos y para BGN en $48,3 \%$. Mientras tanto para los casos de meningitis, este valor de leucocitos se halló en $61,5 \%$ de casos por SGB y $66,7 \%$ de casos por BGN ( $p>0,05)$. En cuanto al LCR, encontramos $5(38,5 \%)$ con meningitis por SGB con leucocitos en LCR cuyos valores oscilaron entre $0-6 / \mathrm{mm}^{3}$, mientras que esto ocurrió en un caso $(16,7 \%)$ de meningitis por BGN. En 11 casos $(84,6 \%)$ de meningitis por SGB la glucosa en LCR estuvo por debajo de $33 \mathrm{mg} / \mathrm{dL}$ y en 5 casos $(83,3 \%)$ de meningitis por BGN. En cuanto a las proteínas en líquido cefalorraquídeo, estas estuvieron por encima de $150 \mathrm{mg} / \mathrm{dL}$ en $8(61,5 \%)$ casos de meningitis por SGB y en $5(83,3 \%)$ de los casos de meningitis por BGN. Las proteínas en LCR se halló más elevado en neonatos con infección por BGN, sin embargo, no hubo diferencia estadísticamente significativa. La PCR se halló significativamente más elevada en los neonatos con sepsis por BGN que por sepsis por SGB. Encontramos valores de PCR por de- bajo de $1 \mathrm{mg} / \mathrm{dL}$ en el 10,5\% de casos de sepsis por BGN y en ningún caso de meningitis por BGN, y en $23,1 \%$ de meningitis y $40 \%$ de sepsis por SGB. No se halló diferencia estadísticamente significativa para hiperbilirrubinemia y acidosis metabólica, pero si para hiperglicemia $\geq 126 \mathrm{mg} / \mathrm{dL}$, la cual estuvo presente en $12,2 \%$ de neonatos con infecciones por BGN y ninguno por SGB, tabla 2 .

La prevalencia de meningitis asociado a sepsis temprana y tardía para SGB fue más elevada $(33,3 \%$; IC 95\%: 14,8-51,9\%) que para sepsis por BGN (4,9\%; IC 95\%: 0,6-16,5\%), diferencia que fue estadísticamente significativa $(\mathrm{p}<0,05)$, tabla 3 . La razón de prevalencia de la enfermedad para la presencia de meningitis entre neonatos con sepsis por SGB y por BGN fue 6,8 (IC 95\%: 1,6-28,9), hallando una asociación estadísticamente significativa entre sepsis y meningitis en neonatos con sepsis por SGB (OR: 9,8; IC 95\%: 1,9-48,8).

En las primeras $72 \mathrm{~h}$ de vida, ocurrieron 2 $(3,1 \%)$ casos de meningitis en sepsis por SGB y $2(4,5 \%)$ casos de meningitis en sepsis por BGN. En neonatos mayores de $72 \mathrm{~h}$ con sepsis 
por SGB ocurrieron $8(24,2 \%)$ casos de meningitis y ningún caso de meningitis en sepsis por BGN, tabla 4. No se halló diferencia en la prevalencia de meningitis asociada a sepsis temprana para SGB y BGN, pero si en la pre- valencia de meningitis asociado a sepsis tardía para SGB al compararlo con sepsis por BGN, con una razón de prevalencia de exposición de 3,6 (IC 95\%: 2-6,2; p < 0,001).

Se encontró un 3\% de muerte entre los

Tabla 2. Manifestaciones clínicas y hallazgos de laboratorio

\begin{tabular}{|c|c|c|c|c|}
\hline Variable & Medida & SGB $n: 30$ & BGN n: 41 & $\mathbf{P}$ \\
\hline $\begin{array}{l}\text { Manifestaciones clínicas } \\
\text { Fiebre } \\
\text { Fiebre persistente } \\
\text { Hipotermia } \\
\text { Gastrointestinales } \\
\text { Respiratorias } \\
\text { Cardio-hemodinámicas } \\
\text { Neurológicas } \\
\text { Afectivas } \\
\text { Ictericia }\end{array}$ & $\begin{array}{l}\text { n (\%) } \\
\text { n (\%) } \\
\text { n (\%) } \\
\text { n (\%) } \\
\text { n (\%) } \\
\text { n (\%) } \\
\text { n (\%) } \\
\text { n (\%) } \\
\text { n (\%) }\end{array}$ & $\begin{array}{l}25(83,3 \%) \\
19(63,3 \%) \\
6(20 \%) \\
16(53,3 \%) \\
17(56,7 \%) \\
12(40 \%) \\
12(40 \%) \\
18(60 \%) \\
6(20 \%)\end{array}$ & $\begin{array}{r}24(58,5 \%) \\
34(82,9 \%) \\
4 \quad(9,8 \%) \\
20(48,8 \%) \\
10(24,4 \%) \\
10(24,4 \%) \\
13(31,7 \%) \\
15(36,6 \%) \\
8(19,5 \%)\end{array}$ & $\begin{array}{c}0,026 \\
0,061 \\
0,220 \\
0,705 \\
<0,01 \\
0,160 \\
0,470 \\
0,051 \\
0,960\end{array}$ \\
\hline $\begin{array}{l}\text { Laboratorio hemograma } \\
\text { Leucocitos en sangre } / \mathrm{mm}^{3} \\
\text { Neutrofilos en sangre/mm³ } \\
\text { Plaquetas en sangre } / \mathrm{mm}^{3} \\
\text { Proteína C reactiva }(\mathrm{mg} / \mathrm{dL})\end{array}$ & $\begin{array}{l}\text { Promedio (DE) } \\
\text { Promedio (DE) } \\
\text { Promedio (DE) } \\
\text { Mediana (RI) }\end{array}$ & $\begin{array}{c}11.832( \pm 8.704) \\
7.716( \pm 6915) \\
366.007( \pm 212.116) \\
3(0,5-7)\end{array}$ & $\begin{array}{c}14.364( \pm 7.338) \\
9.174( \pm 5.989) \\
274.775( \pm 146.312) \\
8,3(3,1-14,8)\end{array}$ & $\begin{aligned} & 0,192 \\
& 0,349 \\
& 0,982 \\
&< 0,01\end{aligned}$ \\
\hline $\begin{array}{l}\text { Líquido cefalorraquídeo } \\
\text { Número de leucocitos } \\
\text { Número de polimorfonucleares } \\
\text { Glucosa } \\
\text { Proteínas }\end{array}$ & $\begin{array}{l}\text { Promedio (DE) } \\
\text { Promedio (DE) } \\
\text { Promedio (DE) } \\
\text { Promedio (DE) }\end{array}$ & $\begin{array}{c}217,5( \pm 902) \\
189( \pm 802) \\
44,2( \pm 24,8) \\
244,2( \pm 147,1)\end{array}$ & $\begin{array}{c}344( \pm 1823) \\
343( \pm 1822) \\
51,2( \pm 20) \\
63,2( \pm 12,5)\end{array}$ & $\begin{array}{l}0,747 \\
0,689 \\
0,258 \\
0,209\end{array}$ \\
\hline $\begin{array}{l}\text { Otros laboratorios } \\
\text { Hiperbilirrubinemia } \\
\text { Hiperglicemia ( } \geq 126 \mathrm{mg} / \mathrm{dL} \text { ) } \\
\text { Acidosis metabólica }\end{array}$ & $\begin{array}{l}N(\%) \\
N(\%) \\
N(\%)\end{array}$ & $\begin{array}{l}7(23,3 \%) \\
0 \quad(0 \%) \\
6(20 \%)\end{array}$ & $\begin{array}{l}8(19,5 \%) \\
5(12,2 \%) \\
5(12,2 \%)\end{array}$ & $\begin{array}{l}0,697 \\
0,047 \\
0,369\end{array}$ \\
\hline
\end{tabular}

SGB: Streptococcus del grupo B. BGN: Bacteria Gram negativas. DE: Desviación estándar. RI: Rango intercuartil. Manifestaciones clínicas: Fiebre: temperatura $>37,5^{\circ} \mathrm{C}$. Fiebre persistente: 2 o más alzas térmicas $>37,5^{\circ} \mathrm{C}$ en $12 \mathrm{~h}$. Hipotermia: Temperatura menor de $36^{\circ} \mathrm{C}$. Gastrointestinales: Hiporexia, vomito, distensión de abdomen, retorno bilioso por sonda, etc. Respiratorias: Taqui o bradipnea, apnea, dificultad respiratoria (quejido, tirajes intercostales, retracción subcostal, aleteo nasal, disbalance toraco-abdominal. Cardio-hemodinámicas: Taqui o bradicardia, mala perfusión, hipotensión. Neurológicas: Compromiso de sensorio, letargo, hipoactividad, convulsiones, focalización. Afectivas: Irritabilidad, Ilanto débil, llanto quejumbroso.

Tabla 3. Distribución de la sepsis y meningitis temprana y tardía

\begin{tabular}{|c|c|c|c|c|c|c|}
\hline Variable & Medida & $\begin{array}{c}\text { SGB } \\
n: \mathbf{3 0}\end{array}$ & $\begin{array}{l}\text { BGN } \\
n: 41\end{array}$ & $\begin{array}{l}\text { Prevalencia de } \\
\text { meningitis en } \\
\text { sepsis por SGB }\end{array}$ & $\begin{array}{l}\text { Prevalencia de } \\
\text { meningitis en } \\
\text { sepsis por BGN }\end{array}$ & $\mathbf{p}$ \\
\hline Sepsis temprana & n (\%) & $11(33,3 \%)$ & $16(36,4 \%)$ & - & - & - \\
\hline $\begin{array}{l}\text { Meningitis asociada a } \\
\text { sepsis temprana }\end{array}$ & $\mathrm{n}(\%)$ & $2(3,1 \%)$ & $2(4,5 \%)$ & $15,4 \%(1,9-45,4 \%)$ & $11,1 \%(1,4-34,7 \%)$ & 0,726 \\
\hline Sepsis tardía & $\mathrm{n}(\%)$ & $9(27,3 \%)$ & $23(50 \%)$ & - & - & - \\
\hline $\begin{array}{l}\text { Meningitis asociada a } \\
\text { sepsis tardía }\end{array}$ & $\mathrm{n}(\%)$ & $8(24,2 \%)$ & 0 & $47,1 \%(23-72,2 \%)$ & 0 & $<0,001$ \\
\hline $\begin{array}{l}\text { Meningitis asociada a } \\
\text { sepsis temprana y tardía }\end{array}$ & n (\%) & $10(33,3 \%)$ & $2(4,9 \%)$ & $33,3 \%(14,8-51,9 \%)$ & $4,9 \%(0,6-16,9 \%)$ & $p<0,01$ \\
\hline
\end{tabular}

SGB: Streptococcus del grupo B. BGN: Bacteria Gram negativas. 
Tabla 4. Aislamientos bacterianos

\begin{tabular}{|lcrrr|}
\hline Bacteria & Sangre & $\begin{array}{c}\text { Sangre y } \\
\text { LCR }\end{array}$ & $\begin{array}{c}\text { Orina y } \\
\text { sangre }\end{array}$ \\
\hline SGB & $20(50 \%)$ & $10(83,3 \%)$ & 0 \\
\hline Escherichia coli & $9(22,5 \%)$ & 0 & $17(89,5 \%)$ \\
\hline Enterobacter spp & $3(7,5 \%)$ & $1(8,3 \%)$ & $2(10,5 \%)$ \\
\hline Serratia spp & $3(7,5 \%)$ & 0 & 0 \\
Klebsiellas spp & $1(2,5 \%)$ & $1(8,3 \%)$ & 0 \\
Seudomona spp & 2 & $(5 \%)$ & 0 & 0 \\
\hline Salmonella spp & 2 & $(5 \%)$ & 0 & 0 \\
\hline Total & $40(100 \%)$ & $12(100 \%)$ & $19(100 \%)$ \\
\hline
\end{tabular}

SGB: Streptococcus del grupo B.

neonatos con infección por SGB y ocurrieron $3(6,8 \%)$ para los Gram negativos ( $\mathrm{p}>0,05)$. Entre las BGN, la $E$. coli fue la predominante, seguido por Enterobacter spp. A pesar que la $E$. coli fue la BGN predominante en sangre, esta no se asoció a meningitis, tabla 4 .

\section{Discusión}

En este trabajo encontramos que una baja paridad, presencia de factores de riesgo para sepsis neonatal por SGB agrupados (parto prematuro, antecedente de hermano con sepsis por SGB, madre febril o con corioamnionitis, madre con ruptura prolongada de membranas) y el parto por vía vaginal, se hallaron asociados a sepsis y meningitis por SGB, factores que ya han sido descritos en la literatura ${ }^{13-15}$. No se halló diferencia entre la edad al momento de la infección para SGB y BGN, pero sí una asociación entre sepsis tardía por SGB y meningitis. Llama la atención que la mayoría de los casos, tanto de sepsis por SGB, como por BGN fueron tardías. Esto apoya el hecho que no se ha demostrado que las estrategias preventivas disminuyan la incidencia de sepsis tardía por SGB, como tampoco la denominada muy tardía sobre los 3 meses de edad ${ }^{15}$.

En cuanto a las manifestaciones clínicas no se halló diferencia significativa entre SGB y BGN. Sin embargo, hallamos una mayor proporción de signos clínicos en neonatos con infección por SGB, en quienes de manera significativa fueron más frecuentes la fiebre y las manifestaciones respiratorias. Para Jain y cols., las manifestaciones respiratorias fueron las más frecuentes $(42,6 \%)$ en neonatos con sepsis, seguido por letargia (40\%), ictericia $(30 \%)$, fiebre $(28,3 \%)$, pobre alimentación $(28,3 \%)$ y distensión de abdomen $(26,7 \%)^{16}$. Dado la importante morbilidad y mortalidad por sepsis, a pesar que la clínica es inespecífica, se debe tener un alto índice de sospecha ante cualquier factor de riesgo y manifestación clínica sugestiva de proceso infeccioso ${ }^{17}$.

La bacteremia es un factor de riesgo conocido para meningitis neonata $1^{18-20}$, con incidencias meningitis tan altas como $23 \%{ }^{13}$. Sin embargo, una tercera parte de los casos de meningitis puede ocurrir en ausencia de sep$\operatorname{sis}^{18}$. Por ello, se debe tener en cuenta que el hemocultivo no debe ser usado como criterio único para decidir la necesidad de realización de una punción lumbar, ya que los hemocultivos hasta en un $38 \%$ pueden ser negativos en neonatos con sepsis ${ }^{13}$. La punción lumbar debe ser realizada en neonatos con hemocultivos positivos, en neonatos cuyo curso clínico o de laboratorio sea fuertemente sugestivo de una sepsis bacteriana y en neonatos a quienes se les vaya a iniciar terapia antibiótica, pudiendo ser diferida en neonatos con descompensación cardio-respiratoria ${ }^{13}$.

La meningitis por SGB ocurrió en $80 \%$ de los casos en neonatos de más de $72 \mathrm{~h}$ de vida, constituyendo a la sepsis tardía en un riesgo para meningitis por SGB. Ansong y cols., en una serie de casos de sepsis por SGB, describen como la meningitis se presentó en $15 \%$ de neonatos con sepsis temprana y $57 \%$ con sepsis tardía ${ }^{19}$. Puede ocurrir una superposición de la presentación clínica inicial de la enfermedad y la de aparición tardía. La enfermedad de inicio tardío se puede presentar hasta en un $24 \%$ como meningitis en comparación con sólo el $6 \%$ de la enfermedad de inicio temprano ${ }^{21}$. En nuestra unidad a todos los casos de sospecha de sepsis tardía, a todos los neonatos febriles, con signos neurológicos y con hemocultivos positivos, se les realiza punción lumbar. En nuestra cohorte, el 33,3\% de las sepsis por SGB se asociaron a meningitis, mientras que esto ocurrió en el 4,9\% de las sepsis por BGN, ningu- 
no de ellos por E. coli. Para Ansong y cols., la meningitis fue complicación en 22/145 (15\%) de los episodios de sepsis temprana por SGB y en 13/23 (57\%) de los episodios de sepsis tardía por esta bacteria ${ }^{19}$, siendo el SGB la causa más frecuente de meningitis ${ }^{20,22}$. La meningitis por BGN se ha asociado con bacteremia, parto prematuro, sexo masculino, defectos del tubo neural y anomalías del tracto urinario ${ }^{23-25}$. En nuestro trabajo, no hallamos que las BGN, parto prematuro, varones e infección urinaria asociada a sepsis, tuvieran un riesgo aumentado o se asociaran con meningitis.

Es cierto que el diagnóstico definitivo de la sepsis y la meningitis, requiere como condición el aislamiento de la bacteria en sangre y líquido cefalorraquídeo ${ }^{11,12,26}$. Sin embargo, en el enfoque diagnostico nos apoyamos en laboratorios que, como el hemograma y los reactantes de fase aguda pueden ser útiles. Uno de los problemas del hemograma es su baja sensibilidad, que puede llegar a ser menor de $50 \%{ }^{27,28}$. Para nuestro caso, hallamos valores de leucocitos, neutrófilos y plaquetas que se encuentran dentro de la normalidad, a pesar que para los neonatos con sepsis por BGN hubo valores más elevados de leucocitos y neutrófilos, cuando se compara con el hemograma de neonatos con SGB. Estos datos concuerdan con los de Villegas y cols., para quienes las BGN tuvieron leucocitos más elevados (mediana 14.700/

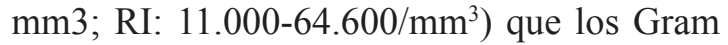
positivos (mediana $13.700 / \mathrm{mm}^{3}$; RI: $2.500-$ $74.000)^{29}$. Actualmente se recomienda la combinación de los varios componentes hematológicos en escalas, sin embargo, estas alcanzan una modesta mejoría en la especificidad y el valor predictivo positivo, sin una reducción en la sensibilidad ${ }^{26}$.

En cuanto a los reactantes de fase aguda, la PCR representa una herramienta importante junto con las manifestaciones clínicas para aquellos casos de sepsis con hemocultivo negativo, clasificados hoy día como sepsis probable. Al igual que el hemograma, la sensibilidad está entre $29-90 \%$ en los diferentes trabajos $^{30-32}$. De ahí, que la determinación seriada de la PCR, la primera al momento de la evaluación del neonato y otra a las 12 a 24 h, mejora la sensibilidad a un $92 \%$ en bacteremias por BGN y $64 \%$ en bacteremias por Gram positivos $^{26}$. En nuestro trabajo, hallamos una diferencia significativa entre la mediana de la PCR, con una valor más bajo para SGB que para BGN $(\mathrm{p}<0,05)$.

Para el LCR, con excepción de la cantidad de proteínas que fueron significativamente más bajas en neonatos con meningitis por BGN, los demás componentes no mostraron diferencias. Este hallazgo difiere de Smith y cols. ${ }^{33}$, para quienes hubo mayor número de leucocitos en LCR de neonatos con meningitis por BGN (mediana: 1.217/ $\mathrm{mm}^{3}$; RI: 43-3.263/ $\mathrm{mm}^{3}$ ), cuando se compara con neonatos con meningitis por cocos Gram positivos (mediana: 187 ; RI: 38-980/mm3), diferencia que fue estadísticamente significativa $(p<0,05)$. No hallaron diferencias para niveles de glucosa (BGN, mediana $22 \mathrm{mg} / \mathrm{dL}$; RI: 9-52 mg/dL. Cocos Gram positivos, mediana: $36 \mathrm{mg} / \mathrm{dL}$; RI: 3-55 mg/dL), proteínas (BGN, mediana 996 mg/dL; RI: 171-588 mg/dL. Cocos Gram positivos, mediana: $279 \mathrm{mg} / \mathrm{dL}$; RI: 146-550 $\mathrm{mg} / \mathrm{dL}),(\mathrm{p}>0,05)$.

Nuestro trabajo no identificó diferencia para hiperbilirrubinemia, ni acidosis metabólica, pero si para hiperglicemia, entre neonatos con sepsis y meningitis por SGB y por BGN, siendo esta última más frecuente en recién nacidos con infecciones por BGN. La hiperglicemia y la acidosis metabólica están asociados con sepsis con una relativa buena capacidad predictiva (área bajo ROC: 0,75$)^{34}$.

La meningitis neonatal por BGN ha sido asociada con aumento de la mortalidad (30$41 \%$ ) cuando se compara con meningitis por cocos Gram positivos ${ }^{18,35}$. En nuestra serie hubo cuatro muertes, una ocurrida para SGB y tres para BGN. Smith y cols. ${ }^{32}$, no encontraron diferencias en mortalidad por tipo de bacteria (coco Gram positivo y BGN). La mortalidad por meningitis por SGB alcanza un $30 \%$, mientras un $50 \%$ de los que sobreviven tienen secuelas neurólogicas que incluyen hidrocéfalia, ceguera, sordera y discapacidades en el neurodesarrollo ${ }^{36}$.

Existen varias limitaciones de este estudio, como el pequeño tamaño de la muestra, afectando el poder para hallar diferencias en algunas variables medidas entre los dos grupos 
comparados. Por otro lado, dado que la sensibilidad de los hemocultivos no es del 100\%, un número de niños con sepsis probables se perdieron, ya que sólo los recién nacidos con cultivos en sangre positivos fueron incluidos en el estudio.

En conclusión, en esta experiencia, la meningitis fue más frecuente en neonatos con sepsis por SGB, principalmente en casos de sepsis tardía. La asociación entre sepsis y meningitis en BGN fue menos frecuente para las presentaciones tempranas y no se presentó en sepsis tardía. Dada la elevada mortalidad y el daño neurológico en los sobrevivientes de neonatos con meningitis, sumado a la prevalencia de meningitis asociada a sepsis encontrada en nuestro trabajo, hacemos énfasis en la importancia de la realización de la punción lumbar en todos los neonatos con sepsis, tanto temprana como tardía, ya que el diagnóstico de infección meníngea puede modificar el esquema y tiempo terapéutico, lo que a su vez afecta el pronóstico final.

\section{Referencias}

1.- Bizzarro MJ, Raskind C, Baltimore RS, Gallagher $P G$ : Seventy-five years of neonatal sepsis at Yale: 1928-2003. Pediatrics 2005; 116 (3): 595-602.

2.- Bizzarro MJ, Dembry L-M, Baltimore RS, Gallagher $P G$ : Changing patterns in neonatal Escherichia coli sepsis and ampicillin resistance in the era of intrapartum antibiotic prophylaxis. Pediatrics 2008; 121 (4): 689-96.

3.- Gibbs RS, Schrag S, Schuchat A: Perinatal Infections due to Group B Streptococci. Obstet Gynecol 2004; 104: 1062-76.

4.- Mendoza LA, Arias M: Susceptibilidad antimicrobiana en una Unidad de Cuidados Intensivos Neonatales: experiencia de 43 meses. Revista de Enfermedades Infecciosas en Pediatría. 2010; 23 (93): 13-24.

5.- Stoll BJ, Hansen N, Fanaroff AA, et al: Changes in pathogens causing early-onset sepsis in very-low-birthweight infants. N Engl J Med 2002; 347 (4): 240-7.

6.- Cordero L, Rau R, Taylor D, Ayers LW: Enteric Gramnegative bacilli bloodstream infections: 17 years' experience in a neonatal intensive care unit. Am J Infect Control 2004; 32 (4): 189-95.

7.- Daley AJ, Isaacs D: and the Australasian Study Group for Neonatal Infections. Ten-year study on the effect of intrapartum antibiotic prophylaxis on early onset group B streptococcal and Escherichia coli neonatal sepsis in Australasia. Pediatr Infect Dis J 2004; 23 (7): 630-4.

8.- Schrag SJ, Zell ER, Lynfield R, et al: A populationbased comparison of strategies to prevent early-onset group B streptococcal disease in neonates. N Engl J Med 2002; 347 (4): 233-9.

9.- Jeeva S, Ramesh A, Ashok D, Vinod P: Sepsis in the newborn. Indian J Pediatr 2008; 75 (3): 261-6.

10.- Muller-Pebody B, Johnson AP, Heath PT, Gilbert RE, Henderson KL, Sharland M: iCAP Group (Improving Antibiotic Prescribing in Primary Care). Empirical treatment of neonatal sepsis: are the current guidelines adequate? Arch Dis Child Fetal Neonatal Ed 2011; 96: F4-F8.

11.- Haque K: Definitions of bloodstream infection in the newborn. Pediatr Crit Care Med 2005; 6 [Suppl.]: S45S49.

12.- Overturf $G$ : Defining bacterial meningitis and other infections of the central nervous system. Pediatr Crit Care Med 2005; 6 [Suppl.]: S14-S18.

13.- Polin R: Management of Neonates With Suspected or Proven Early-Onset Bacterial Sepsis. Pediatrics 2012; 129 (5): 1006-15.

14.- Schrag S, Gorwitz R, Fultz-Butts K, Schuchat A: Prevention of perinatal group B streptococcal disease. Revised Guidelines from CDC. MMWR Recomm Rep 2002 (rr-11): 1-22.

15.- Cruz M, Doren A, Tapia JL, Abarzúa F: Sepsis neonatal por Streptococcus del Grupo B. Rev Chil Pediatr 2008; 79 (5): 462-70.

16.- Jain NK, Jain VM, Maheshwari S: Clinical Profile of Neonatal Sepsis. Kathmandu University Medical Journal 2003; 1 (2): 117-20.

17.- Gerdes JS: Diagnosis and management of bacterial infections in the neonate. Pediatr Clin North Am 2004; 51: 939-59.

18.- Stoll BJ, Hansen N, Fanaroff C, et al: To tap or not to tap: high likelihood of meningitis without sepsis among very low birth weight infants. Pediatrics 2004; 113 (5): 1181-6.

19.- Ansong A, Smith PB, Benjamin D, et al: Group B streptococcal meningitis: cerebrospinal fluid parameters in the era of intrapartum antibiotic prophylaxis. Early Hum Dev 2009; 85 (10 suppl): S5-S7.

20.- Garges HP, Moody A, Cotten CM, et al: Neonatal meningitis: what is the correlation among cerebrospinal fluid cultures, blood cultures, and cerebrospinal fluid parameters? Pediatrics 2006; 117 (4): 1094-100.

21.- Schrag SJ, Zywicki S, Farley MM, et al: Group B 
streptococcal disease in the era of intrapartum antibiotic prophylaxis. N Engl J Med 2000; 342: 15-20.

22.- Wiswell TE, Baumgart S, Gannon CM, Spitzer AR: No lumbar puncture in the evaluation for early neonatal sepsis: will meningitis be missed? Pediatrics 1995; 95 (6): 803-6.

23.- Mangi RJ, Quintiliani R, Andriole VT: Gram-negative bacillary meningitis. Am J Med 1975; 59: 829-36.

24.- Unhanand M, Mustafa MM, McCracken Jr GH, Nelson JD: Gram-negative enteric bacillary meningitis: a twenty-one-year experience. J Pediatr 1993; 122: 15-21.

25.- Mulder CJ, van Alphen L, Zanen HC: Neonatal meningitis caused by Escherichia coli in The Netherlands. J Infect Dis 1984; 150: 935-40.

26.- Benitz $W$ : Adjunct Laboratory Tests in the Diagnosis of Early-Onset Neonatal Sepsis. Clin Perinatol 2010; 37 : 421-38.

27.- Manroe BL, Weinberg AG, Rosenfeld CR, et al: The neonatal blood count in health and disease. I. Reference values for neutrophilic cells. J Pediatr 1979; 95 (1): 8998.

28.- Rodwell RL, Leslie AL, Tudehope DI: Early diagnosis of neonatal sepsis using a hematologic scoring system. J Pediatr 1988; 112 (5): 761-7.

29.- Villegas R, Muro R, Garduño J, Cuevas M, Madrigal O, Estrada J, García H: Diagnóstico etiológico de sepsis neonatal basado en factores de riesgo e índices hemato- lógicos. Enf Inf Microbiol 2008; 28 (2): 51-9.

30.- Fowlie PW, Schmidt B: Diagnostic tests for bacterial infection from birth to 90 days-a systematic review. Arch Dis Child Fetal Neonatal Ed 1998; 78 (2): F928.

31.- Dollner H, Vatten L, Austgulen R: Early diagnostic markers for neonatal sepsis: comparing $\mathrm{C}$-reactive protein, interleukin-6, soluble tumour necrosis factor receptors and soluble adhesion molecules. J Clin Epidemiol 2001; 54 (12): 1251-7.

32.- Garland SM, Bowman ED: Reappraisal of C-reactive protein as a screening tool for neonatal sepsis. Pathology 2003; 35 (3): 240-3.

33.- Smith PB, Cotten CM, Garges HP, et al: A comparison of neonatal Gram-negative rod and Gram-positive cocci meningitis J Perinatol 2006; 26: 111-4.

34.- Griffin M, Lake D, Moorman R: Heart Rate Characteristics and Laboratory Tests in Neonatal Sepsis. Pediatrics 2005; 115: 937-41.

35.- Sarman G, Moise AA, Edwards MS: Meningeal inflammation in neonatal Gram-negative bacteremia. Pediatr Infect Dis J 1995; 14: 701-4.

36.- Leib SL, Kim YS, Chow LL, Sheldon RA, Täuber MG: Reactive oxygen intermediates contribute to necrotic and apoptotic neuronal injury in an infant rat model of bacterial meningitis due to Group B streptococci. J Clin Invest 1996; 98 (11): 2632-9. 